\title{
Extracellular Matrix Components and Integrins in the Control of Arterial Smooth Muscle Cell Structure and Function
}

\author{
Ulf Hedin \\ Department of Experimental Surgery and Department of Surgery, Vascular Division, Karolinska Hospital, \\ Stockholm, Sweden.
}

During the first few days of primary culture, smooth muscle cells from the aortic media of adult rats modulate from a quiescent, contractile state to a secretory active and proliferative state, synthetic phenotype. This phenotypic modulation seems to be a prerequisite for smooth muscle cell proliferation both in vitro, in atherogenesis and during the development of neointimal hyperplasia after reconstructive vascular surgery. Because the extracellular matrix influence cellular function in many different experimental situations, we have explored the ability of matrix molecules to modulate the phenotypic properties of cultured rat arterial smooth muscle cells in order to evaluate if changes in the pericellular matrix may take part in the control of smooth muscle phenotype and proliferation in vascular disease.

Below, a summary of recent results from our research is presented. For further reading, a review by Thyberg et al. (1) is recommended. Taken together, these results show that extracellular matrix components, by receptormediated mechanisms, are capable of inducing changes in smooth muscle cell structure, expression and organization of contractile proteins, and function. This indicates a close relationship between integrin-mediated cell-matrix interactions, cytoskeletal organization and the control of smooth muscle cell phenotype which may be important during smooth muscle cell migration and proliferation in atherogenesis.

\section{Structure and function of arterial smooth muscle cells in atherogenesis}

In the adult organism, the arterial media is composed of highly differentiated smooth muscle cells (SMCs) arranged in concentric layers. The individual cells are encircled by an incomplete basement membrane composed of type IV collagen, laminin, entactin, and heparan-sulfate proteoglycans. Most likely, this extracellular matrix regu-

This article was presented at the Winter 1991 meeting of the Japan Atherosclerosis Society in Tokyo. lates the cellular homeostasis by direct interactions, transport of molecules and by providing a link with surrounding tissue. The latter primarily consists of elastin, type I and III collagen, and chondroitin- and dermatan sulfate proteoglycans. Ultrastructurally, adult SMCs are characterized by a cytoplasm filled with myofilaments, abundant mitochondria, and few synthetic organelles. The cytoskeleton contains myosin and tropomyosin as well as a SMC-specific isoform of $\alpha$-actin (SMC $\alpha$-actin). Functionally, they contract in response to stimulation with vasoactive hormones but the basal synthesis of proteins and DNA is low. Evidently, this contractile phenotype is not in a stable differentiated state. In atherogenesis and in neointimal hyperplasia, fibroblast-like SMCs are found in the lesion. These cells have few myofilaments in the cytoplasm, the endoplasmic reticulum and the Golgi complex are prominent, and there is an ongoing cell

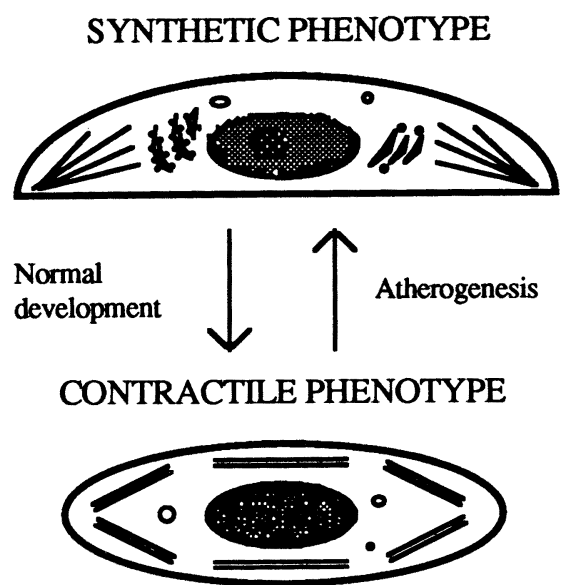

Fig. 1. Phenotypic modulation of SMCs occurs in normal development as the proliferating SMCs of growing vessels differentiate into contractile cells. In atherogenesis, SMCs are believed to return back to a primitive, synthetic phenotype. 
proliferation. The cytoskeleton is dominated by nonmuscle actin isoforms such as $\beta$ - and $\gamma$-actin and the expression of myosin is lower than in the contractile cells of the vascular media. Thus, a transition, or modulation, of the cells from a quiescent, contractile state to a synthetic, proliferative phenotype has been assumed to occur in the initial phase of the atherosclerosis process. This synthetic state represents a partial dedifferentiation of the cells back to a primitive, fibroblast-like, phenotype normally found in the arterial media of embryos and growing organisms (Fig. 1; Thyberg et al. (1)).

\section{Effect of extracellular matrix components on fine struc- ture and function of cultured SMCs}

When enzymatically isolated SMCs are placed in primary culture, a similar transition from contractile to synthetic cells occurs as in atherosclerosis. Morphologically, this process has the same features as described above, and functionally it results in an activation of synthetic activities and cell proliferation. Because the structural modification of the cells precedes cell replication, the phenotypic modulation has been recognized as a

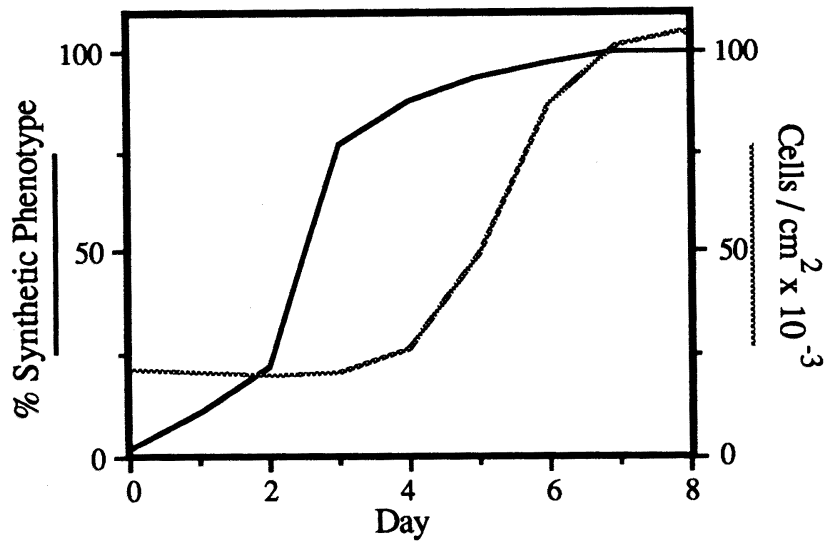

Fig. 2. Phenotypic modulation and proliferation of arterial smooth muscle cells during primary culture in serum-containing medium. Phenotypic state of the cells was evaluated by electron microscopy and proliferation by trypsinization and cell counting in a haemocytometer. Note the shift in phenotypic state of the cells prior to the proliferative response.

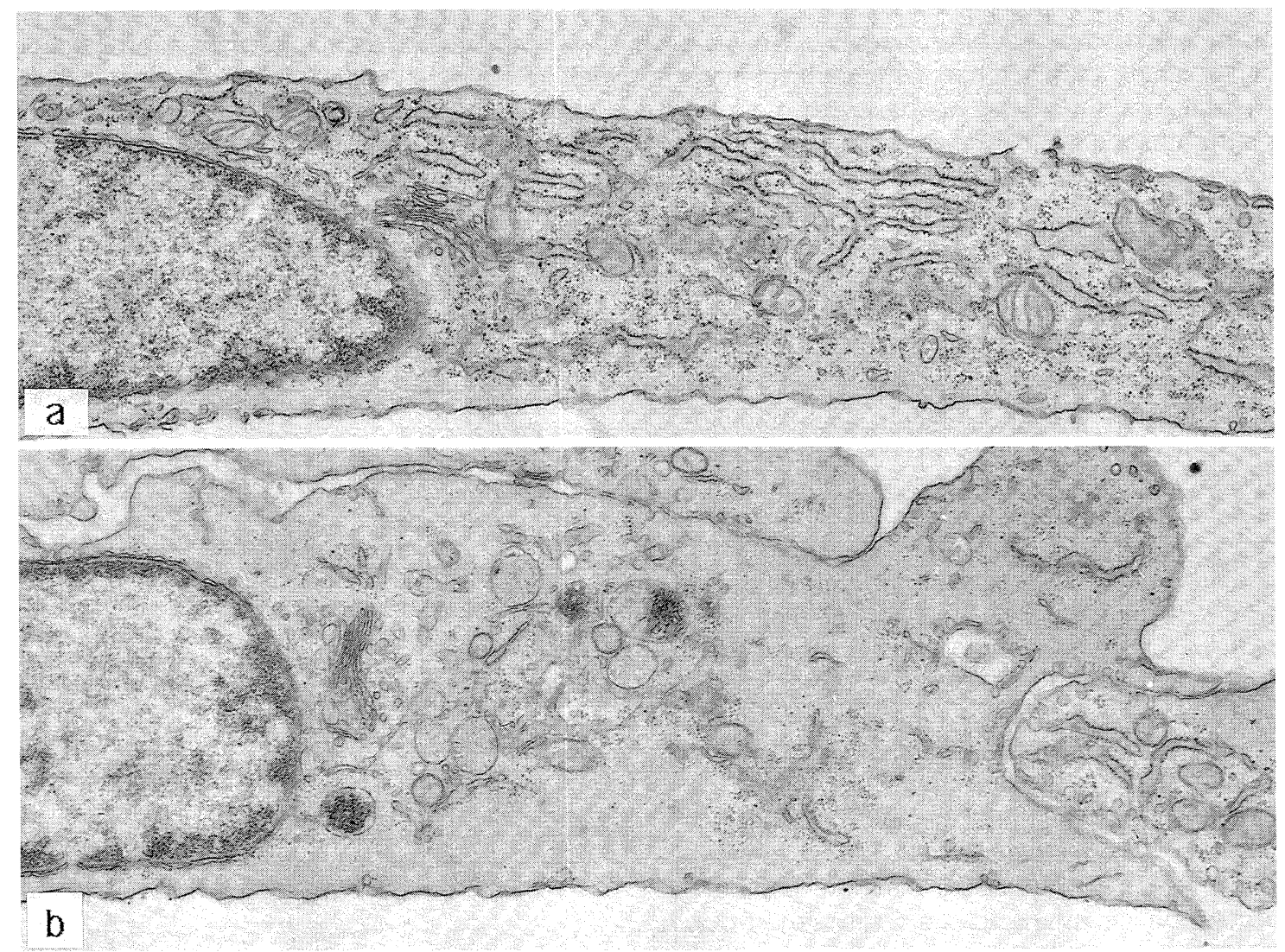

Fig. 3. Fine structure of SMCs on a substrate of FN (a) and laminin (b). The cells were isolated by collagenase digestion of the rat aortic media and cultured on the substrates in serum-free medium. The cultures were fixed after 2 (a) and 6 (b) days of culture and prepared for electron microscopy. Cells cultured on FN have modulated into a synthetic phenotype (a) whereas cells on laminin remain contractile in the presence of soluble RGD-peptide (b). 
prerequisite for the ability of the cells to respond to growth factor stimulation (Fig. 2). Since we experienced that this process took place equally well in the absence of serum mitogens, we assumed that other serum components might influence the phenotypic state of cultured SMCs. By investigating different serum fractions, we found that addition of plasma fibronectin ( $\mathrm{pFN}$ ) to serum-free medium efficiently promoted the phenotypic modulation during the first days of primary culture. This plasma- and extracellular matrix protein was even more potent when prepared as a substrate on the bottom of the petri dish. If seeded in medium $\mathrm{F}-12 / 0.1 \%$ BSA on a substrate of $\mathrm{pFN}$, freshly isolated rat SMCs rapidly attach and spread out with an plating efficiency of $60-80 \%$. After 2-4 days of culture, most cells have lost their contractile appearance as evaluated by the electron microscopy. The cytoplasm have a low volume fraction of myofilaments and there is a large endoplasmic reticulum and Golgi complex (Fig. 3a; Hedin and Thyberg (2)). Functionally, there is an activation of RNA- and protein synthesis, and the cells start to secret extracellular matrix components. However, the cells remain quiescent and DNA-synthesis and cell proliferation will not start until exogenous mitogen is added to the culture medium. (Hedin and Thyberg (2), Hedin et al. (3)).

Because $\mathrm{FN}$ is a constituent of the extracellular matrix we investigated the effects of other matrix components on the modulation process. Vitronectin (VN) was able to promote the phenotypic modulation of cultured SMCs in a similar manner as FN but at a slower rate (unpublished observation). On the other hand, the basement membrane proteins type IV collagen and laminin, closely asociated with SMCs in the normal vascular media, was shown to have strikingly different effects on the phenotypic properties of SMCs. On these substrates,

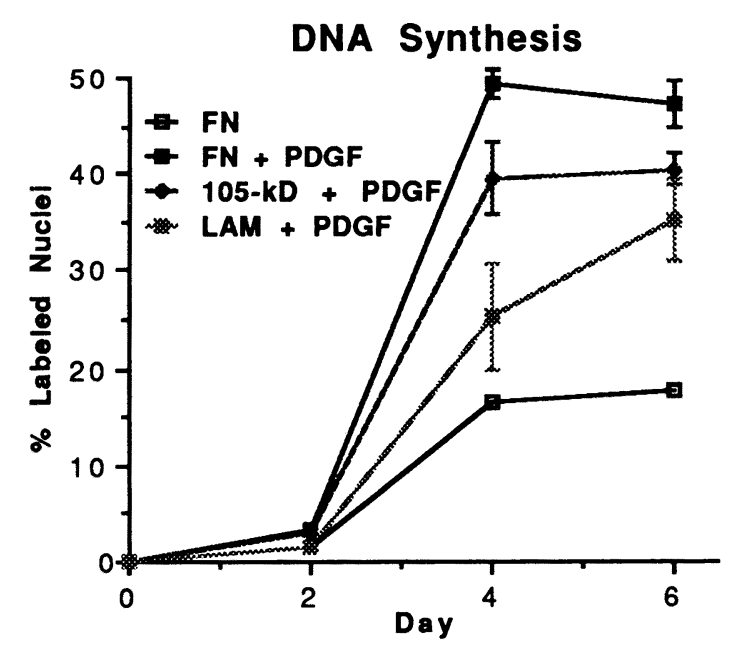

Fig. 4. Induction of DNA synthesis in SMCs during primary culture on FN, a cell binding fragment of FN (105-kD), and laminin (LAM). The cultures were stimulated with PDGF and DNA-synthesis determined by ${ }^{3} \mathrm{H}$-thymidine autoradiography.

\section{CONTRACTILE PHENOTYPE}

\section{SYNTHETIC PHENOTYPE}

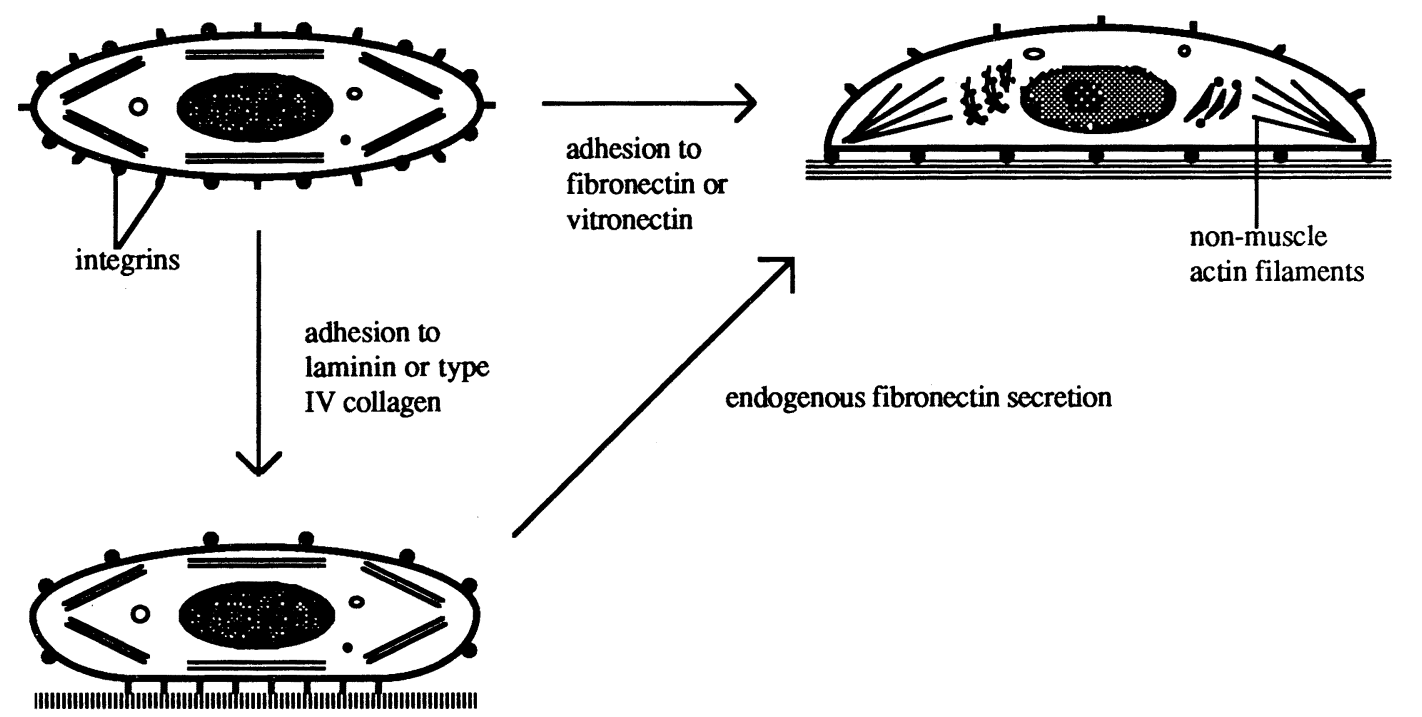

Fig. 5. Scematic illustration of the different effects of FN, laminin and type IV collagen on the phenotypic properties of SMCs during primary culture. On FN, there is rapid attachment and spreading of the cells followed by a transition into a synthetic phenotype. On basement membrane substrates, the contractile phenotype is retained and a delayed spreading and modulation occurs due to endogenous secretion of FN. 
attachment and cell spreading was less efficient than on FN. The cells slowly spread in a spindle-shaped manner and the ultrastructural characteristics of a contractile phenotype was retained for 3-5 days (Fig. 3b). Moreover, DNA-synthesis and cell proliferation was lower after stimulation with PDGF (platelet-derived growth factor) in cells cultured on laminin than in cells cultured on FN (Fig. 4). After 4-6 days, cell spreading on laminin and type IV collagen was enhanced and there was a shift from a contractile to a synthetic phenotype. Since this process could be blocked both by RGD-peptides (Arg-Gly-Asp) which bind to the $\mathrm{FN}$ receptor $\alpha_{5} \beta_{1}$, the delayed phenotypic modulation on laminin and type IV collagen was assumed to occur due to endogenous secretion of FN. In fact, cells cultured on laminin and type IV collagen was shown to secrete more FN than cells cultured on FN-derived substrates, indicating that the cells may sense their molecular environment and adjust the production of matrix components accordingly (Fig. 5 ; Hedin et al. (3)).

In addition to these traditional matrix proteins, we have also studied the effects of thrombospondin and tenascin on the behaviour of cultured SMCs. Neither of these proteins are capable of promoting efficient spreading of freshly isolated SMCs. They were both secreted and incorporated in the pericellular matrix after 2-3 days of culture. Tenascin was absent in the carotid and aortic media of adult rats but accumulated in the neointima formed two weeks after balloon catheter injury to the carotid artery. In addition, tenascin was found to be secreted in association with the phenotypic modulation occurring during primary culture in serum-free medium on FN. The expression of tenascin by SMCs seems to be associated with the modulation process both in vivo and in vitro but the role of this protein in the regulation of SMC phenotype and proliferation is still unknown (Hedin et al. (4)).

\section{Interactions involved in the phenotypic modulation of SMCs on FN}

By using chymotryptic fragments of pFN, the ability of FN to promote the modulation process was shown to reside in the cell-binding domain of the molecule. This was further investigated by seeding freshly isolated SMCs on RGD-containing peptide substrates where the modulation process occurred as efficiently as on intact FN. Furthermore, this process was suppressed to a similar extent on FN- and RGD-substrates by antibodies to the $\beta_{1}$ subunit of the fibronectin receptor. This indicated that the RGD-substrate was able to promote the change in phenotype of the cells by interacting with a $\beta_{1}$-containing FN-receptor on the cell surface. To verify if SMCs were able to spread completely on the RGD-peptide, we examined its ability to provoke organization of actin filament bundles in the cytoplasm. This process was as efficient on the RGD-substrate as on intact fibronectin, and independent of ongoing protein synthesis. This corroborated that the RGD-substrate was in fact able to establish functional contacts with the cells (Hedin et al. (5)).

The results described above pointed to the presence of an RGD-dependent FN receptor on the surface of SMCs. This receptor was isolated by sequential affinity chromatography and shown to be composed of two subunits with electrophoretic mobilities in SDS-polyacrylamide gels of 160 and $115 \mathrm{kD}$ under non-reducing conditions and 150 and $130 \mathrm{kD}$ under reducing conditions. Immunochemical and immunocytochemical studies have indicated that these subunits correspond to the $\alpha_{5}$ - and the $\beta_{1}$-subunits of the integrin VLA 5 in SMCs (Akiyama et al. (6), Hemler, 1990). However, a fraction of the two proteins was not eluted from the column with the RGDcontaining peptide, indicating the presence of additional FN-binding, non-RGD dependent, receptors, possibly VLA 3 (Fig. 6 ; Bottger et al. (7)).

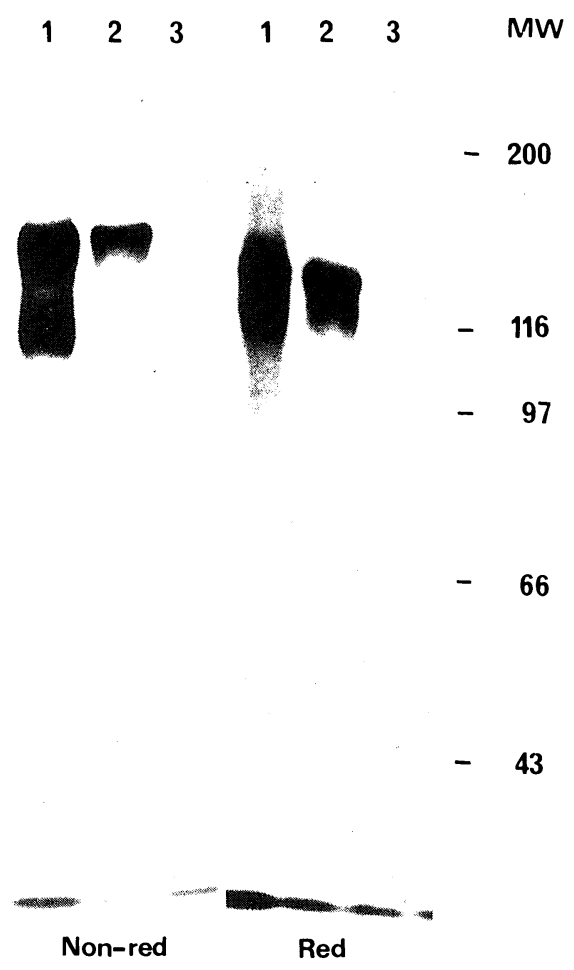

Fig. 6. SDS-polyacrylamide gel electrophoresis of proteins isolated from surface labeled SMCs, subjected to affinity chromatography on WGA-Sepharose followed by chromatography on a column of the cell-binding domain of FN linked to Sepharose. Proteins were eluted with RGD-peptide (lane 1), followed by elution with EDTA (lane 2), and urea (lane 3). Note the presence of non-RGD dependent material left on the column after RGD-elution (lane 2). 
Table 1. Expression of SMC $\alpha$-actin mRNA and non-muscle actin related to phenotype of SMCs during culture on different matrix components in primary culture and subcultivated cells. Actin expression was evaluated by isolation of mRNA and Northern hybridization with specific cDNA probes.

\begin{tabular}{llcc}
\hline \multicolumn{1}{c}{ Cell type } & \multicolumn{1}{c}{ Morphology } & $\beta$-actin mRNA & $\alpha$-actin mRNA \\
\hline Freshly isolated & Contractile & + & H \\
Primary Culture & Synthetic & + & + \\
FN 4d & Synthetic & + & - \\
Serum 6d & & & \\
Subcultivated & Synthetic & + & + \\
FN 2d & Synthetic/Contractile & + & \\
Lam/Coll IV 2d &
\end{tabular}

Taken together, these results indicated that the phenotypic modulation of freshly isolated SMCs on FN was mediated by an interaction between the RGDsequence in the cell-binding domain of $\mathrm{FN}$ and integrin $\alpha_{5} \beta_{1}$ on the cell surface. Immunochemical studies have revealed that this integrin as well as other members of the $\beta_{1}$-integrin family are expressed in the rat aortic media and recent reports have also documented the presence of a collagen- and laminin-binding $\alpha_{1} \beta_{1}$ integrin in SMCs, both in vivo and in vitro (Belkin et al. (8), Clyman et al. (9)).

\section{Role of cytoskeletal reorganization in the phenotypic modulation of SMCs on FN}

In fibroblastic cells, the extracellular matrix have been found to participate in the organization of the actin cytoskeleton, and transmembrane receptor complexes have been implicated in this function (Burridge et al. (10), Woods and Couchman(11)). The spreading and phenotypic modulation of SMCs on a substrate of FN is associated with organization of actin filaments in stress fibers. To further investigate how extracellular matrix components affect SMC phenotype, we analyzed the expression and organization of SMC $\alpha$-actin in freshly isolated SMCs cultured on a substrate of FN under serumfree conditions. Northern blot analysis showed that the expression of mRNA for SMC $\alpha$-actin, but not for nonmuscle actin, was strongly repressed during primary culture (Table 1). Indirect immunofluorescence staining revealed that non-muscle actin was rapidly organized in stress fibers which did not stain with a monoclonal antibody against SMC $\alpha$-actin.

During the first days of culture, filament bundles containing $\alpha$-actin were most prominent in the central parts of the cytoplasm and gradually disappeared as the spreading of the cells progressed (Fig. 7). In contrast to the situation with non-muscle actin, there was no apparent overlap in the staining for $\alpha$-actin and the FN receptor $\left(\alpha_{5} \beta_{1}\right)$, indicating that this receptor interacted with nonmuscle actin during the initial spreading process. Thus, the phenotypic modulation of SMCs on FN apparently depends on the ability of FN-binding integrins to promote the organization of non-muscle actin in stress fibres. Possibly, SMC $\alpha$-actin is associated with other matrix
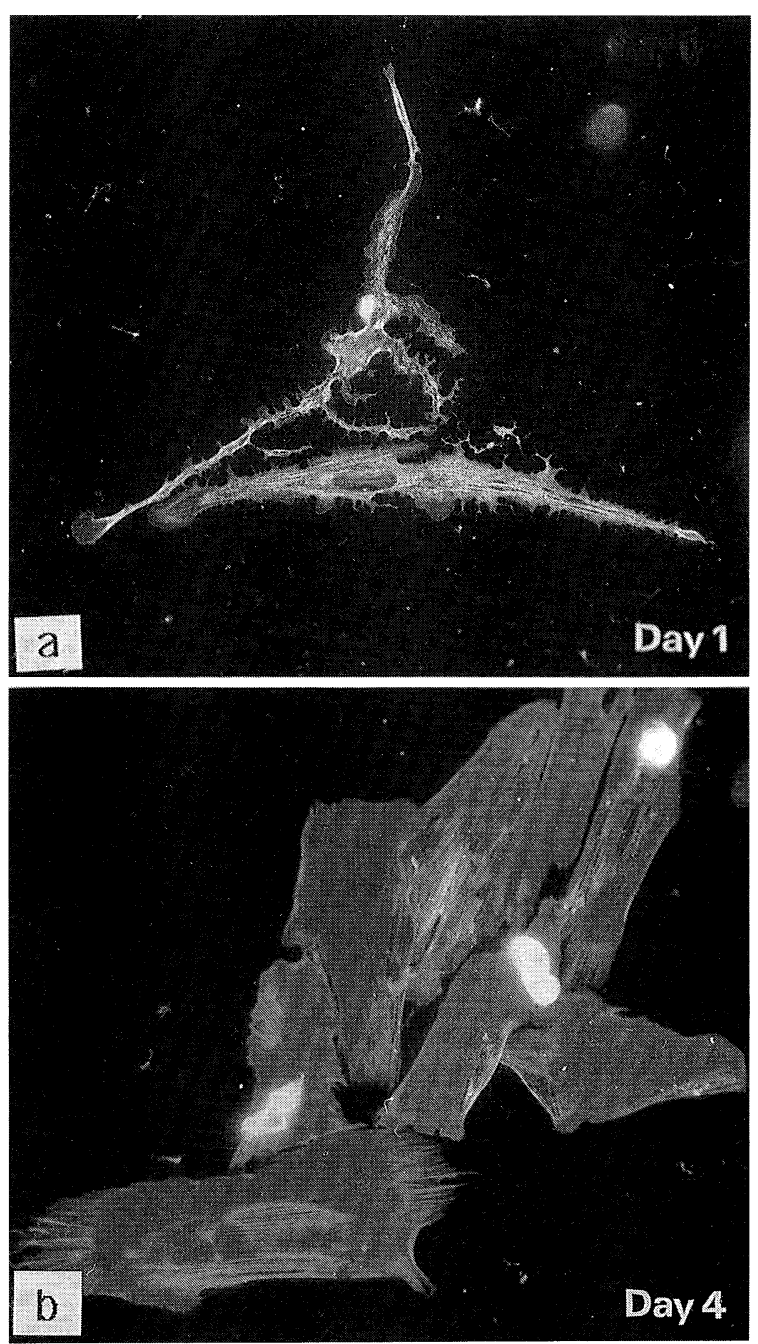

Fig. 7. Immunocytochemical staining for SMC $\alpha$-actin in SMCs cultured under serum-free conditions on a substrate of $\mathrm{FN}$ in primary culture. After 1 day of culture (a), $\alpha$-actin filament bundles occupy large portions of the cytoplasm whereas most staining has disappeared after 4 days of culture (b). 

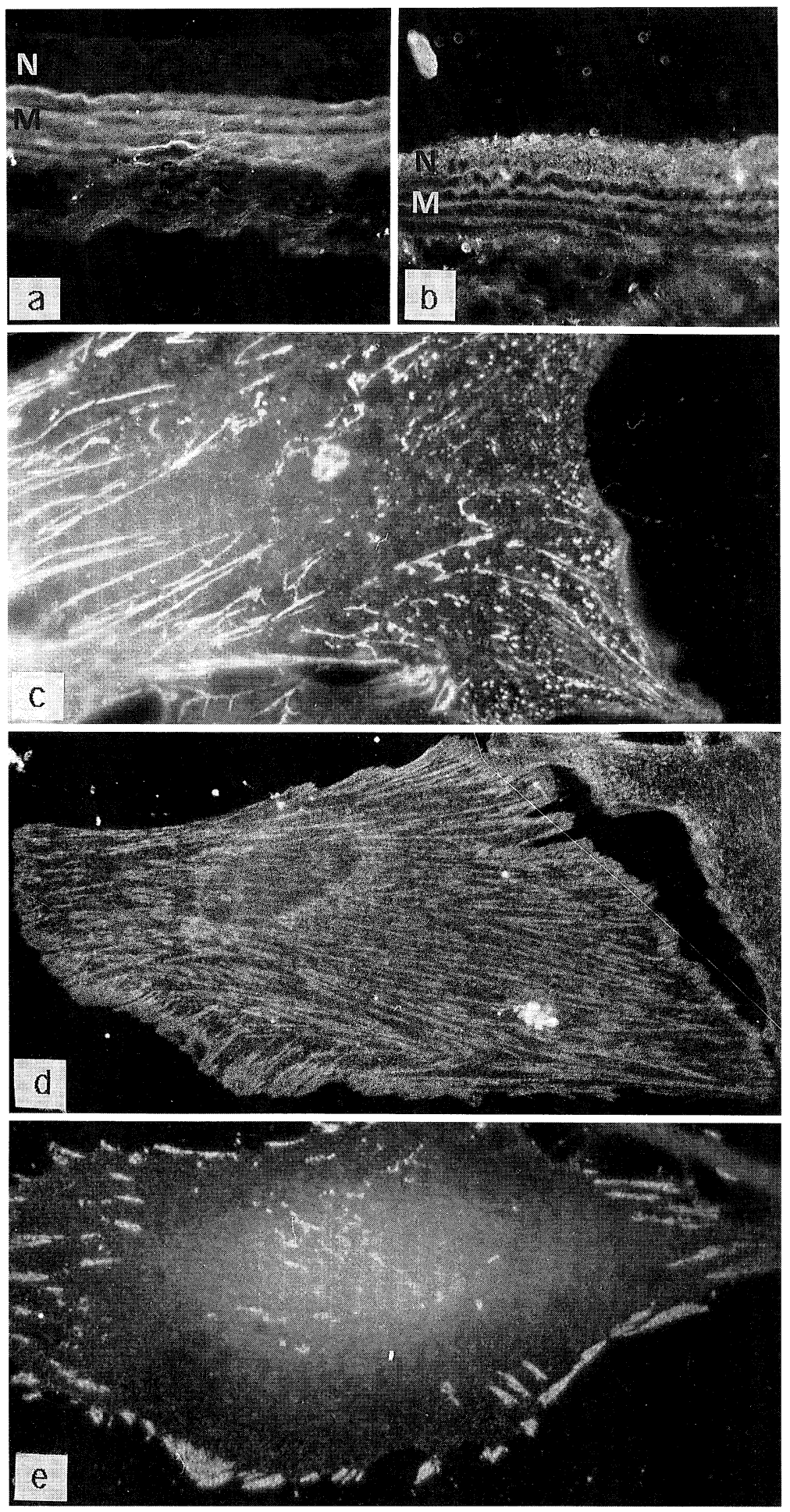

Fig. 8. Immunocytochemical staining for integrin subunits in the media $(\mathrm{M})$ and neointima $(\mathrm{N})$ of balloon injured rat carotid arteries (a and b) and primary SMCs (c-e) on FN substrates in the absence ( $c$ and d) or presence of serum (e). $\quad \alpha_{5}$ (a and $c$ ) is found in the arterial media and in fibrillar arrays on the surface of cultured cells whereas $\alpha_{\mathrm{v}}$ (b and e) is localized in the neointima and in adhesion plaques of cells growing in serum. Note the wide and different distribution of the $B_{1}$ subunit (d) in cultured SMCs as compared with the $\alpha_{5}$ subunit. 
receptors in the contractile cell which may be important for the control of the phenotypic state of the cells (Hedin et al. (12)).

Recent studies have confirmed the idea that extracellular matrix components influence SMC phenotype and expression of $\alpha$-actin in SMCs. If subcultured, synthetic cells are trypsinized and reseeded on different matrices in serum-free medium, the expression of SMC $\alpha$-actin mRNA is increased and there is structural evidence of a partial 'remodulation' to a contractile phenotype in cells seeded on laminin and type IV collagen. In contrast, cells seeded on FN have a decreased expression of $\alpha$-actin mRNA and the cells remain synthetic (Thyberg, unpublished data; Table 1).

\section{Role of integrins in the proliferation of SMCs in vivo and in vitro}

The results described above demonstrate the importance of integrin-mediated interactions between SMCs and the extracellular matrix, cytoskeletal organization, and the control of SMC phenotype in vitro. Nevertheless, it is difficult to predict the biological significance of these results with respect to the changes in SMC structure and function occurring in atherosclerosis. We have examined the expression of the FN receptor $\alpha_{5} \beta_{1}$ by immunohistochemistry in rat carotid arteries after balloon catheter injury. This integrin was expressed in the normal arterial media but no staining could be detected in the neointima (Fig. 8a). In contrast, antibodies against the $\mathrm{VN}$ receptor, $\alpha_{\mathrm{V}} \beta_{3}$, stained the neointima but not the arterial media (Fig. 8b). During primary culture on FN substrates, there is an initial staining for $\alpha_{5}$ in focal adhesions in the cell periphery, indicating that this receptor is involved in the primary adhesion and spreading of the cells. However, after a few days of culture in serumfree medium, this receptor is localized in fibrillar arrays on the cell surface rather than in adhesion plaques (Fig. 8c). This is in contrast with the staining for the $\beta_{1}$ subunit which is found both in adhesion plaques and in a fibrillar pattern along the cell surface (Fig. 8d). This suggests that the RGD-dependent FN receptor $\alpha_{5} \beta_{1}$ is primarily involved in the organization of FN fibrils in the pericellular matrix and that other FN-binding integrins, possibly $\alpha_{3} \beta_{1}$, participates in cell adhesion and cytoskeletal organization in synthetic, proliferative cells. In cell transformation, this receptor seems to be associated with a migrating and proliferative phenotype (Plantefaber and Hynes, 1989), whereas $\alpha_{5} \beta_{1}$ has been observed to participate in matrix organization on the surface of human fibroblasts (Roman et al. (13)). Moreover, the $\mathrm{VN}$ receptor $\alpha_{\mathrm{V}} \beta_{3}$ is almost exclusively found in adhesion plaques of SMCs proliferating in serum where the $\mathrm{FN}$ receptor is localized along the cell surface (Fig. 8e). In human SMCs, Belkin et al. (8) have shown a high expression of the laminin-binding integrin, $\alpha_{1} \beta_{1}$, in contractile cells in vivo whereas the expression disappears during culture. This suggests a close association between the contractile phenotype and basement membrane proteins in agreement with our previous findings. Taken together, these results indicate that the expression of integrins is altered as the phenotypic state of the cells is changed both in vivo and in vitro and that different matrix receptors may have distinct functions as related to SMC proliferation.

\section{Concluding remarks}

The results of our studies show that extracellular matrix components have distinct effects on the phenotypic behaviour of cultured SMCs. The role of individual matrix proteins and receptors is gradually emerging and several observations point to mechanisms where these molecules exert their effects through interactions with cytoskeletal components. Most likely, similar interactions take place in atherogenesis. To investigate the molecular mechanisms involved in phenotype control of contractile SMCs in the normal arterial media and the specific changes occurring in such interactions during migration and proliferation of SMCs in atherogenesis remains an exciting challenge for the future.

Aknowledgements: The main part of these studies have been performed at the laboratory of Assistant Professor Johan Thyberg at the Department of Medical Cell Biology, Karolinska Institutet, Stockholm, Sweden. Financial support was obtained from the Swedish Medical Research Council, the Swedish Society of Medicine, the Swedish Heart Lung Foundation, the Magnus Bergvall Foundation, the Lars Hierta Memorial Fund, and the Tore Nilsson Foundation.

\section{References}

(1) Thyberg J, Hedin U, Sjölund M, Palmberg L, and Bottger BA : Regulation of differentiated properties and proliferation of arterial smooth muscle cells. Arteriosclerosis, 10: 966-990, 1990

(2) Hedin U and Thyberg $\mathrm{J}$ : Plasma fibronectin promotes modulation of arterial smooth-muscle cells from contractile to synthetic phenotype. Differentiation, 33:239246, 1987

(3) Hedin U, Bottger BA, Forsberg E, Johansson S, and Thyberg $\mathrm{J}$ : Diverse effects of fibronectin and laminin on phenotypic properties of cultivated arterial smooth muscle cells. J Cell Biol, 107 : 307-319, 1988

(4) Hedin U, Holm J, and Hansson GK : Induction of tenascin in rat arterial injury: relationship to altered smooth muscle cell phenotype. Am J Pathol, 139: 649-656, 1991

(5) Hedin U, Bottger BA, Luthman J, Johansson S, and Thyberg $\mathrm{J}$ : A substrate of the cell attachment sequence of fibronectin (Arg-Gly-Asp-Ser) is sufficient to promote transition of arterial smooth muscle cells from contractile to synthetic phenotype. Dev Biol, 133: 489-501, 1989

(6) Akiyama SK, Nagata K, and Yamada KM : Cell surface receptors for extracellular matrix proteins. Biochem 
Biophys Acta, 1031 : 91-110, 1990

(7) Bottger BA, Hedin U, Johansson S, and Thyberg J: Integrin-type fibronectin receptors of rat arterial smooth muscle cells : isolation, partial characterization and role in cytoskeletal organization and control of differentiated properties. Differentiation, 41: 158-167, 1989

(8) Belkin VM, Belkin AM, and Koteliansky VE: Human smooth muscle VLA-1 integrin: purification, substrate specificity, localization in aorta, and expression during development. J Cell Biol, 111 : 2159-2170, 1990

(9) Clyman RI, Turner DC, and Kramer RH: An $\alpha_{1} \beta_{1}$-like integrin receptor on rat aortic smooth muscle cells mediate adhesion to laminin and collagen types I and IV. Arteriosclerosis, 10: 402-409, 1990

(10) Burridge K, Fath K, Kelly T, Nuckolls G, and Turner C : Focal adhesions, transmembrane junctions between the extracellular matrix and the cytoskeleton. Ann Rev Cell
Biol, 4 : 487-525, 1988

(11) Woods A and Couchman JR: Focal adhesions and cell-matrix interactions. Collagen Relat Res, 8: 155182,1988

(12) Hedin U, Sjölund M, Hultgårdh-Nilsson A, and Thyberg $\mathrm{J}$ : Changes in expression and organization of smoothmuscle-specific $\alpha$-actin during fibronectin-mediated modulation of arterial smooth muscle cell phenotype. Differentiation, 44: 222-231, 1990

(13) Roman J, LaChance RM, Broekelmann TJ, Kennedy CJR, Wayner EA, Carter WG, and McDonald JM: The fibronectin receptor is organized by extracellular matrix fibronectin: implications for oncogenic transformation and for cell recognition of fibronectin matrices. J Cell Biol, 108 : 2529-2543, 1989 